\title{
Addressing the Future Burden of Cancer and Its Impact on the Oncology Workforce: Where Is Cancer Prevention and Control?
}

\author{
Shine Chang • Carrie Cameron
}

Published online: 25 February 2012

(C) Springer Science+Business Media, LLC 2012

\begin{abstract}
The need for cancer professionals has never been more urgent than it is today. Reports project serious shortages by 2020 of oncology health care providers. Although many plans have been proposed, no role for prevention has been described. In response, a 2-day symposium was held in 2009 at The University of Texas MD Anderson Cancer Center to capture the current status of the cancer prevention workforce and begin to identify gaps in the workforce. Five working groups were organized around the following topic areas: (a) health policy and advocacy; (b) translation to the community; (c) integrating cancer prevention into clinical practice; (d) health services infrastructure and economics; and (e) discovery, research, and technology. Along with specific recommendations on these topics, the working groups identified two additional major themes: the difficulty of defining areas within the field (including barriers to communication) and lack of sufficient funding. These interdependent issues synergistically impede progress in preventing cancer; they are explored in detail in this synthesis, and recommendations for actions to address them are presented. Progress in cancer prevention should be a major national and international goal. To achieve this goal, ensuring the health of the workforce in cancer prevention and control is imperative.
\end{abstract}

Keywords Training $\cdot$ Education $\cdot$ Preparation $\cdot$ Evaluation

S. Chang $(\bowtie) \cdot$ C. Cameron

Department of Epidemiology,

Division of Cancer Prevention and Control,

The University of Texas MD Anderson Cancer Center,

1155 Pressler St., Unit 1365,

Houston, TX 77230-1439, USA

e-mail: ShineChang@MDAnderson.org

\section{Cancer Prevention: Where Are We Now?}

In warning the public about a projected critical shortage of medical oncologists in the USA by 2020 [1], the 2007 study commissioned by the American Society for Clinical Oncology (ASCO) stimulated consideration of the unrecognized potential for cancer prevention efforts by its exclusion from the report. It also raised concern that the field of cancer prevention and control was at risk for a similar workforce shortage in the future [2]. The ASCO report described factors that contribute to the impending medical oncology shortage. These include growth in the number of Baby Boomers at increased risk for cancer, in the number of oncologists eligible for retirement, and in the number of cancer survivors with long-term care needs, as well as the decline in the number of physicians choosing careers in oncology [1]. Using statistical models to test various workforce strategies, ASCO reported that given time and resource constraints for preparing oncologists, simply training more individuals would not address the shortage projected for 2020. Clearly, raising concern regarding the workforce that will manage the future burden of cancer was not misguided.

Since 2007, much has taken place. Soliciting recommendations to address the shortfall, ASCO created and approved a strategic plan in 2008 [3]. Some aspects of ASCO's threegoal plan have already been implemented. These include creating a workforce advisory committee [3], providing grant awards to study workforce issues in partnership with Susan G. Komen for the Cure Foundation [4], conducting pilot projects to test innovative practice models and demonstration projects to build partnerships between oncologists and physician extenders [5], and submitting a statement to the US Senate Health, Education, Labor, and Pensions Committee hearing on health-care workforce initiatives [6]. In spite of these steps to address the oncology workforce 
shortage, major opportunities to achieve progress against cancer through prevention continue to be missed, as most reports do not consider the impact of cancer prevention activities. Such omission may result from lack of awareness, skepticism of the impact of prevention, or the dearth of information available for describing the infrastructure of cancer prevention and control and its workforce [2]. Regardless, if cancer prevention activities are to alleviate the future burden of cancer on our society, their visibility must be raised.

\section{Lessons from Public Health and Medicine}

More than a decade ago, concerns were raised about threats to the public health workforce [7]. Many of these threats impeded description of the public health workforce. A multidisciplinary field that involves many different types of workers, public health comprises formally trained professionals to individuals trained on-the-job, making identification of the individual job descriptions within the workforce difficult [8]. Furthermore, public health activities span basic research to applied practice and take place in a variety of settings (e.g., schools, communities, hospitals, and universities) at different levels (i.e., local, state, and national). They also involve numerous partners such as governmental agencies, non-profit foundations, universities, health-care organizations, private sector partners, and professional membership societies. The field of public health has struggled to create a universal, clearly defined identity for itself, with common language, goals, and standards. Metrics and infrastructure for workforce surveillance and forecasting were lacking, which stimulated enumeration efforts [9]. Since then, important progress has been made. Motivated by research agendas set for studying the public health workforce (Table 1) [8, 10], a body of literature now exists that has benefited over time from improved coordination of efforts (e.g., Office of Workforce and Career Development within Office of the Centers for Disease Control Director), creation of common definitions, surveillance of workforce data (e.g., HRSA), development of resources, methods and models for forecasting and evaluating the public health workforce, including for policy analysis, as well as

Table 1 Public health workforce research themes and annotations [adapted from Gotway Crawford et al. [8]]

1. Workforce size and composition

2. Workforce diversity

3. Workforce effectiveness and health impact

4. Recruitment, retention, separation, and retirement

5. Worker pay, promotion, performance, and job satisfaction

6. Demand for the public health workforce

7. Education, training, and credentialing the public health workforce

8. Public health workforce policy
Measuring, monitoring, and forecasting the workforce size and composition is required for assessing status and historical trends in workforce size and distribution by age, sex, race/ ethnicity, education, job type, geographical distribution, and experience; and for forecasting workforce needs, mandating minimal staffing, and allocation of resources. A national, standardized, and routine surveillance system for classifying and tracking the workforce should be implemented for use with models to identify ideal distribution of the workforce by venue.

To cultivate positive environments that enhance delivery of public health services, workforce surveillance data should also include workforce demographic information that facilitates accommodation of demographic shifts in the workforce and the public when projecting future workforce demands.

A workforce surveillance system should include data and indicators that measure the impact of the workforce on specific aspects of the public's health. Other efforts should include long-term evaluation of training programs and consider the value of credentialing on increasing the quality and performance of the workforce. Methods need to be developed to evaluate the effectiveness of workforce policies and strategies to improve the workforce and its impact on achieving measureable public health outcomes.

Developing the strongest workforce relies on understanding which recruitment and retention strategies are most successful, monitoring trends and key determinants of recruitment and retention in public health organizations, and knowledge of factors that influence decisionmaking in choosing careers in public health.

In addition to needing greater knowledge of the impact of individual-level benefits, organizational practices, climate, and culture and values that are common to all industries and fields, additional information is needed about factors that influence satisfaction and stress specific to professionals in public health.

Methods are needed to measure to the workforce capacity (e.g., size, capability, training, and resources) at different levels, both currently and in the future, as well as workforce models that take into account changes that may influence characteristics and composition of the workforce as well.

Identifying measureable core competencies in public health practice has been proposed to increase individual competency and build workforce capacity, as well as serve as a standard for training the public health workforce. Certification programs may increase knowledge, skills, abilities, and competencies, which would also add value and has been implemented in other disciplines and fields.

To ensure the sustained impact and effectiveness of the public health workforce, assessing the role and impact of policies and strategies to improve and change it is critical. 
dissemination and translation of findings into effective changes [8]. These challenges to clear characterization of the public health workforce are similar to those facing cancer prevention and control today.

Farther along in understanding its workforce, medicine has maintained a rigorous infrastructure for workforce surveillance through several organizations, including the American Medical Association, the Association of American Medical Colleges (AAMC), and the Accreditation Council for Graduate Medical Education. For example, AAMC has maintained national databases of medical schools, applicants, students, graduates, and faculty since 1966 . They have described these resources in terms of their racial and ethnic diversity, gender, student admissions, and faculty recruitment, retention, salaries, and promotions. The ability of the AAMC to analyze available data collected annually from more than 130 medical schools across the USA and Canada has resulted in a rich literature documenting the health of its workforce from a variety of perspectives that has kept attention focused on anticipated workforce shortages in medicine.

Far from being distinct from medicine and public health, cancer prevention and control shares goals and professionals with both. However, cancer prevention has unique features as well. It extends beyond the primary interest of public health, the health of populations, to include focus upon individual patients, like medicine. In contrast to public health and medicine, the field of cancer prevention is specific in its mission to a single disease around which its stakeholders can concentrate their efforts, whereas stakeholders in public health and medicine more often express their support for specific aspects of public health (e.g., vaccinations) or affiliation with specific types of practitioners (e.g., pediatricians), rather than to the broader missions of public health and medicine at large. Medicine has articulated competencies that serve as the basis for all training, board certification, and licensing across the USA. For the cancer workforce, C-Change, a non-profit organization oriented to eliminating and preventing cancer, has created "Cancer Core Competencies" [11, 12], as part of a larger initiative to address cancer workforce issues, but these competencies are not widely recognized within the field. Thus, while much remains to be done in both public health and medicine, the field of cancer prevention and control can profit from advances made in these and other fields to understand and address issues faced by its own workforce.

\section{"The Future Directions in Cancer Prevention and Control: Workforce Implications for Training, Practice, and Policy" Symposium}

In 2009, a group of cancer prevention and control professionals organized a 2-day symposium at The University of
Texas MD Anderson Cancer Center, with the objective of capturing the current status of the cancer prevention workforce and beginning to identify gaps in the workforce. Five working groups were organized around topic areas: (a) health policy and advocacy; (b) translation to the community; (c) integrating cancer prevention into clinical practice; (d) health services infrastructure and economics; and (e) discovery, research, and technology. Following the symposium, these groups formed writing groups to develop manuscripts about workforce issues relevant to the topic areas. Trainees attending the symposium through sponsored scholarships helped facilitate writing groups and collaborated to produce a meeting report [13]. A group addressing the perspective of nurses in cancer prevention was added later, and the health policy and advocacy group was reorganized to write the article included in this supplement. In total, six manuscripts are included in this supplement together with this synthesis and other materials and information from the symposium. Each article reflects the perspectives and experiences of the authors and their knowledge of the literature and available resources. They are offered here as a means to stimulate dialogue about workforce issues in the field of cancer prevention and control.

\section{Themes from Writing Groups}

In addition to the unique ideas and recommendations presented in each article, two major themes emerged from the writing groups: the difficulty of defining areas within the field (including the barriers to communication and the difficulties created), and the lack of sufficient funding. These interdependent themes, along with their sequelae, synergistically impede progress in preventing cancer.

\section{Definitions in Cancer Prevention and Control}

The field of cancer prevention and control includes a diversity of disciplines from basic sciences to population-based research, such as biostatistics, epidemiology, and behavioral science, as well as a multitude of interventions to be disseminated into community and clinical practice, all of which are united only by its defining goal - to prevent cancer. The research in these disciplines varies substantially, by study design, population focus, or model system (e.g., genetic and molecular factors, cell lines, animal models, and humans), by research methods and tools, and by technical language. Thus, a challenge to professionals within the field is communicating across disciplines, especially when different terms are used to describe similar concepts and when similar terms have different meanings. The challenge is made more complex when collaborations involve professionals from 
disciplines outside the field who have not previously been oriented to the goals or methods of cancer prevention.

Several consequences result from poor communication, one of the most serious being lack of awareness of potential cross-disciplinary collaborations. For example, professionals outside cancer prevention may not realize that their skills and expertise are relevant to cancer prevention and control. Conversely, those working in cancer prevention may not recognize the potential gains from collaborating with those outside the field (or know how to reach them), such as exposure to new approaches and methods and specialized technology, resources, and perspectives that could infuse the field with fresh ideas and energy. Without good communication, those within the field of cancer prevention and control risk isolation and missed opportunity for innovative thinking. Moreover, if information about activities within the field is not communicated externally, risk for wasteful duplication of resources and efforts in cancer prevention is greater. Altogether, these consequences of poor communication slow the pace of discovery and progress within the field.

Poor communication within the field impedes clear and uniform definition of emerging areas of focus, a critical issue for new areas to organize successfully and be nurtured. For such areas of emphasis to thrive, the individuals involved in those areas must be able to identify one another, which may be difficult if the fields are labeled differently (e.g., "cancer prevention health services research" vs. "comparative effectiveness in cancer prevention"). Not knowing about colleagues with similar interests because they describe themselves differently impedes collaborations, development of methods and resources, and generation of interest around the subject matter or topic area. In this era of team science, rare is the occasion when a major breakthrough is achieved in isolation. Without commonly defined goals, individuals may lack orientation to the most critical issues to be addressed, diluting impact, and progress in cancer prevention. This problem may be especially detrimental to those early in their careers because junior scientists may compete more easily for resources in emerging areas than in those with established experts. If it is difficult for junior scientists to identify such emerging areas then they cannot take advantage of them. Clear definitions are also critical for funding agencies to recognize promising new directions for cancer prevention, as well as to support training in such areas. Only once sufficient momentum is gained can more rapid discovery and advancement be possible, and emerging areas become established.

The Cancer Prevention Workforce and Poor Communication

Poor communication affects the cancer prevention workforce in several ways. First, awareness of the career opportunities in the field of cancer prevention is lacking among both students and those who provide career advice to them. Most students are aware of careers in medicine, nursing, and public health practice, as well as of careers in biological, public health, and behavioral science research, but many may not connect these careers with opportunities in cancer prevention. Research on the Millenial generation suggests that young people obtain information about careers and make career decisions differently than previous generations, with a focus on initial searches through the Internet, a preference for "branded" employers and direct contact with decision-makers in target organizations, and a strong preference for individualized, tailored career development assistance. Because students ignorant of career opportunities in cancer prevention will not seek them out, raising the profile of cancer prevention careers is vital. Unfortunately, exposure of health professional students to cancer prevention careers during training remains low. For example, over the past 20 years since efforts by the American Association for Cancer Education have sought to assess and integrate cancer prevention education into undergraduate medical education, and in spite of recommendations by the IOM [13], recent review of medical school curricula suggests only modest success achieved [14]. Improved efforts to raise awareness among medical students could have potent impact, as $57 \%$ of entering oncology fellows surveyed by ASCO reported deciding to pursue oncology before residency [15]. Medical school represents a critical window of opportunity for influencing physician career choices, as oncology competes with other subspecialties for those boarded in internal medicine [16]. Using a variety of strategies, including new methods of communication (e.g., social media), students must be engaged more effectively and exposed to cancer prevention careers earlier in their education.

For those already interested in cancer prevention careers, part of the problem may be structural, in that no clear career path into the field exists for students to follow. For example, no specialty or subspecialty is currently dedicated to providing training in cancer prevention. Medical students hoping to pursue accredited post-graduate medical training relevant to clinical cancer prevention are limited to choosing either preventive medicine specialty, which does not focus exclusively on cancer, or internal medicine subspecialty in oncology, which does not focus exclusively on prevention.

Some learn about cancer prevention later in their careers, sometimes after years of practice in basic science and clinical work in oncology. If knowledge of careers in cancer prevention comes later during the training trajectory, fatigue from lengthy training or lack of interest in returning to status as students or trainees may deter some, even if they are interested in careers in cancer prevention. Moreover, debt accrued from medical training, while not consistently shown to influence choice of specialty [17], may discourage those 
seeking additional training in cancer prevention, especially if it comes after other required career preparation. The National Cancer Institute (NCI) is interested in building the cancer prevention workforce and provides funding for training and career development awards designed to attract professionals into research careers in cancer prevention [18]. While these have been successful and have launched the careers of many researchers, the methods for recruiting individuals into the field are left to free-market competition. At any rate, by not creating highly visible and desirable career paths into cancer prevention and control, we may already be losing excellent individuals.

\section{Insufficient Funding in Cancer Prevention and Control}

A second major barrier to achieving success in cancer prevention and control that the writing groups cited often was insufficient funding. Indeed, in 2010, while the NCI spent $31.7 \%$ for research in cancer causation, detection, and diagnosis and $22.7 \%$ for treatment research, a total of $7.1 \%$ of the NCI annual budget was allocated for cancer prevention and control [19]. This reflects a decreasing trend over time in the proportion of the total budget for funds specifically allocated for cancer prevention and control, which was $10.7 \%$ in $2006,10.4 \%$ in 2007 , and $9.9 \%$ in 2008 before the budget mechanism for cancer prevention and control was eliminated altogether in 2009. Of course, the NCI is not the only source of funding for cancer prevention and control activities. Many other federal agencies and philanthropic organizations, both large and small, contribute to supporting activities to prevent cancer. In spite of these collective efforts, progress in cancer prevention and control, regardless of funds expended, remains moderate at best.

The perception of the writing groups that insufficient funding forms a major barrier to cancer prevention is natural given the breadth of disciplines within the field, and the wide scope of activities and their complexity that fall under the rubric of cancer prevention and control. For research, the ability to pursue more ambitious goals at a faster pace than in past has been facilitated by major advances in technology, including computing, robotics, and faster, easier to use, and omnipresent tools for communication. These have increased the pace of research, but not always decreased its cost. Moreover, discoveries in cancer prevention research that are translated into effective interventions may have associated costs as well, such as for procedures, for tests or assays, or personnel effort and time to deliver educational and behavioral change strategies. Of course, the more intractable that major cancer risk factors are, such as inherited susceptibility to cancer, tobacco use, and obesity, the greater the resources needed to address them successfully may be, but also the more the professional community and the public may want efforts redoubled. Gaining headway against such risk factors may be especially challenging due to the complex etiology of these conditions. Of course, cancer prevention professionals endure constant pressure from the public's desire for easy and low-cost methods to prevent cancer with little or no side-effects.

A long-standing problem to clinical implementation of proven cancer prevention strategies has been limited reimbursement for conducting cancer prevention activities. The proportions of oncologists reporting "insufficient reimbursement for prevention activities in clinical practice" as a barrier to cancer prevention did not change substantially, from $65 \%$ to $63 \%$, in the 15 years between surveys conducted in 1989 and 2004 [20, 21]. Such a report is disappointing in light of the advances in cancer prevention achieved to date. Other advances pose cost-related challenges as well. For example, the exciting results in 2011 demonstrating the effectiveness of CT screening for lung cancer early detection reported from the National Lung Screening Trial [22] raises many cost-related issues about who should be screened and how often, as well as whether the cost of regular screening will be prohibitively expensive and who will pay for it [23]. It is to be expected that policies to reimburse for specific cancer prevention services will always lag behind the development of scientific discoveries into tools that are proven effective; however, the decisionmaking process by which policy makers, patient advocates, insurers, and health-care providers determine which services to include in cancer prevention coverage may need improvement in order to have greater impact on the burden of cancer [13].

\section{Funding for Training the Cancer Prevention Workforce}

Funding for recruiting and preparing the professional workforce in cancer prevention and control exists. In 2010, the NCI allocated 3.5\% of its annual budget $(\$ 179,346,000)$ to research manpower development [19]. This amount encompasses support for all research training in cancer, including for cancer prevention and control scientists. Of the 17 extramural training and educational award mechanisms listed on the NCI's Center for Cancer Training's website, three specifically provide support for individuals and one for institutions to support multiple trainees in cancer prevention research training. The total amount in 2010 for individual awards was \$21.4 M (21 K05 awards at \$3.1 M; $97 \mathrm{~K} 07$ awards at $\$ 13.3 \mathrm{M}$; and $30 \mathrm{~K} 05$ awards at $\$ 5.0 \mathrm{M}$ ) and for the 44 institutional R25T awards, the total amount was \$20.4 M [24], equaling 23.3\% for cancer prevention of the total amount spent by NCI on developing the research workforce. The NCI portfolio comprises only a portion of the support expended in the USA for training and education in cancer prevention and control; other arms of the $\mathrm{NIH}$, other federal agencies (e.g., AHRQ, DOD, and CDC), non-profit foundations (e.g., American Cancer Society, 
Prevent Cancer Foundation, AICR), and even for-profit companies also provide support for training and education in cancer research. In 2001, the American Cancer Society estimated that approximately $20 \%$ of its total spending in training and career development was dedicated to cancer prevention, between \$2 and \$3 M annually [13]. In Texas, the Cancer Prevention and Research Institute of Texas (CPRIT) has funded 350 awards since 2010 for more than $\$ 570 \mathrm{M}$, of which 12 of 27 were in prevention education, for a total of $\$ 5.5 \mathrm{M}$. This amount represents $57 \%$ of the total $\$ 9.7 \mathrm{M}$ awarded for prevention activities or $0.97 \%$ of all CPRIT funds awarded to date [25]. To recruit and retain individuals in cancer careers, a recent partnership between the American Society for Clinical Oncology and the Susan G. Komen for the Cure Foundation provides loan repayment for oncologists from populations underrepresented in medicine committed to serving medically underserved communities [26, 27].

The availability of such funds represent a major commitment to preparing the next generation of cancer professionals; however, it is unclear whether the total amount of funds dedicated to training cancer prevention professionals - either in Texas or across the nation-is sufficient to staff and serve the growing needs for cancer prevention and control, particularly in proportion to other public health issues. Indeed, one indicator that funding may be insufficient comes from the long-standing and alarming steady decline in numbers of physicians serving in public health leadership [13]. Also, residency programs in preventive medicine are shrinking, both in total number, from 90 programs in 1999 to 72 in 2012, and in the annual number of residents in training, down $15 \%$ since 2001 [28, 29], partly because nearly half of available training positions are left unfilled due to insufficient funding [30]. Supporting residency programs in preventive medicine is particularly challenging. Unlike all other residency programs that qualify for support from Medicare Graduate Medical Education because they are hospital based, preventive medicine residency programs do not receive Medicare funding because preventive medicine residents are typically trained outside hospitals [30]. While graduate medical training in both preventive medicine and oncology includes some exposure to cancer prevention and control, overcoming the challenges to funding training in prevention for health-care providers is critical to having sufficient numbers in the cancer prevention and control workforce.

In spite of the funding awarded to date for training in cancer prevention and control, the writing groups opined that too few training programs in cancer prevention and control existed overall, and that too few existed to populate specific areas of need within the field. Indeed, in 1999, the NCI reshaped the focus of its R25T award program in response to its perceived need to populate cancer research with professionals prepared to participate in multidisciplinary research [13]. This award mechanism is well-suited to the types of cross-disciplinary projects and environments that are the norm in cancer prevention and control, and has facilitated a more vigorous effort to recruit researchers into the field. Of the 43 awards active in 2010, 22 allow for research training in a variety of areas within cancer prevention and 11 focus on specific aspects, such as behavioral science, nutrition, genetics, or tobacco [24]. Such programs provide fellowship support for those learning how to conduct research in cancer prevention through basic science, population and behavioral science, and clinical translation. The R25T programs differ from other NIH-funded training programs (e.g., Kirschstein NSRA T32 awards) in that they require trainees to have a minimum of two mentors with complementary expertise to facilitate development of crossdisciplinary research skills (NCI R25T PAR-10-165). Careful nurturing of trainees preparing for such multidisciplinary careers is required if the educational and research experiences provided are not to revert to uni-disciplinary training or to be diluted by countervailing mentoring, institutional, or other environmental factors antagonistic to multidisciplinary approaches [2].

To supplement the recruitment of new cancer prevention researchers, some writing groups described a need to provide supplementary training in cancer prevention to professionals who are already established in their careers, a recommendation espoused in the IOM's report, "Fulfilling the Potential for Cancer Prevention and Early Detection" [13]. Several state cancer plans include providing cancer prevention education for a variety of health-care providers [31]. However, the state cancer plans vary in support received, and the amount of state funding expended for strengthening the cancer prevention workforce and its impact are unknown. Funding is available for workshops that focus on "novel cancer-focused state-ofthe-art research or methodological knowledge, or evidencebased cancer prevention and control interventions" through the NCI R25E award mechanism (PAR-12-049), although funds are not specifically targeted for health-care providers and would focus on them only if proposed to do so by sponsoring institutions. Awards are granted for up to 5-years at $\$ 300,000 /$ year in direct costs and are not renewable, which may limit the long-term impact of cancer prevention training for providers if sponsors are unable to sustain developed programs after NCI funding ends. One cancer prevention education resource available to physicians for purchase was developed by the American Society for Clinical Oncology (ASCO): "Cancer Prevention Curriculum" book and CD ROM, and its maintenance module [32]; offering up to $28.5 \mathrm{~h}$ of CME credit, the book and CD cover a wide range of topics organized into 27 chapters, including tobacco, diet, screening, chemoprevention, community interventions, ethics of clinical trials, survivorship, and 
nine chapters about organ-specific cancers. At its annual meeting, ASCO has created a track to feature research and education in cancer prevention. Oncologists and primary care physicians alike recognize the need to deliver cancer prevention services to their patients [33] as together, they coordinate the long-term care for increasing numbers of cancer survivors and those at risk for cancer. However, even practicing oncologists report a need for more information and training to increase confidence in delivering cancer prevention and control care more effectively to their patients $[20,34]$. Thus, the critical challenge then becomes how to reach ASCO members and beyond to other healthcare providers, to provide training in cancer prevention to improve coordinated care, as well as how to pay for it [1].

\section{Other Issues for Future Consideration}

Critical issues remain for future consideration. For example, in the USA, educators currently struggle to attract students into research careers in general. Negative perceptions of features of science careers such as low salaries, long hours, little recognition, and high competition do little to draw interest. This problem is compounded by lack of awareness of careers opportunities in science, let alone for careers in cancer prevention. Also unknown is the impact of changing preferences in work expectations on recruiting and retaining the future cancer prevention workforce. Gen X, Millennials, and younger generations have different lifestyle and work priorities from their parents and earlier generations, such that new strategies are needed to accommodate generational differences. At the same time, the fierce competition for funding in the sciences demands ever increasing effort from researchers. It is not clear how these conflicting trends can be reconciled.

The academic health science centers environment in which research takes place, including for cancer prevention, is not appealing to many faculty, younger or older, and makes retention and reward additional challenges. Many, even established, faculty struggle to maintain productivity in the face of increasing administrative demands, while accommodating other professional and personal needs. Careers in academia are not widely perceived as enjoyable or worth the effort, and efforts to change the toxic environment are not on the horizon. NIH has made some important changes to allow more individuals to gain recognition for their work in the form of multiple PI-led grant awards, and journals recognize co-first authored articles. However, the ratio of tenure-track positions to those who aspire to them remains problematic. Generational culture change, decreases in funding levels, the paucity of secure faculty positions, and the explosive pace of scientific and technological advances have come together in a sort of perfect storm, dramatically increasing the volume of work to be done while decreasing the number of researchers available. Creative solutions to address these issues will be essential for building and retaining the future cancer prevention workforce.

One important moderator of the trend toward a decreasing workforce is the internationalization of scientific research. Thousands of scientists from around the world are coming to the USA for training and research (as well as working in their home countries) and making substantial contributions to cancer prevention research. The total number of foreign scientists and engineers working in academia in the USA has more than doubled in the past 35 years [35], and non-US citizens have constituted more than half of the US biosciences postdoctoral population since 2004 [36]. The influx of international scientists helps to mitigate decreases in the research workforce. At the same time, however, immigration restrictions, cultural and linguistic barriers in academia, and growth in some foreign economies, such as China's, induce many of these researchers to return to their home countries. But given the projected increases of cancer incidence and mortality worldwide ( $27 \%$ and $45 \%$ according to $\mathrm{WHO}$ ) [37], the need for cancer prevention efforts around the world will be of crucial importance, and cancer prevention efforts must be relevant to the types and causes of cancers found in various geographical regions, infrastructures and economic environments, and cultural landscapes. This is especially true of cancers that are preventable by behavior change, which will require specialized, culturally appropriate interventions. Thus, welltrained researchers in cancer prevention are needed around the world. Future efforts for the cancer prevention workforce must not neglect this critical topic.

\section{The Need for a National Cancer Prevention Workforce Plan}

Some gaps in the cancer prevention and control workforce have already been identified. For example, the National Cancer Registrar Association has reported anecdotal evidence for shortages in the professionals who manage and staff tumor registries that are critical for cancer surveillance. Feedback from a 2006 workforce survey indicated unfilled or difficult to fill registrar positions that vary by type of position and geographic region [38]. Other shortages are more difficult to quantify, but increasing job opportunities in cancer prevention have been reported [2]. To support health science training and education across the country, the NIH has used funding mechanisms that vary in scope and size. Some are broad and support a wide range of disciplines for professional training. Others are narrower in focus, in order to facilitate targeted recruitment of 
professional expertise within specific topics or disciplines. This system has prospered through free-market competition of institutions and individuals.

Benefits of using an open competition system to establish health science training and education are several. They include providing equal opportunity to compete for resources and the freedom to determine how best to address the workforce needs that they perceive, taking advantage of the unique strengths of their sponsoring institutions and leaders. Indeed, minimal requirements imposed by funding agencies allow programs to range in size and approach, maximizing the fit of training programs to particular research niches within the field. Many large health science centers have competed successfully for training program support, taking advantage of greater capacity and existing infrastructure.

Disadvantages to free competition exist as well. For example, unguided proliferation is likely to create a hodgepodge of programs that duplicates efforts and resources, leaving gaps unaddressed. As expanding training activities where programs or expertise already exist does not broaden the workforce where it is needed, funding agencies may be discouraged from supporting new training programs, whether they are of equal quality or better than existing ones. Also, training resources often go to large research centers that have other assets and advantages for sponsoring educational activities, but smaller centers may have greater need to develop expertise. The latter may not compete well, having disadvantages that are difficult to overcome without funding support that then becomes a self-perpetuating problem. This may help explain why several states in the Midwestern United States do not have NCI-designated comprehensive cancer centers or NCI-funded training programs, as gaps may occur either in topic or by geographic region, or both. Another disadvantage is the amount of time needed for training relative to the urgency of addressing shortages. While some professions can train large numbers of individuals quickly for positions that require less education and training [16], other professions that require more intensive preparation may not be able to mobilize sufficiently fast if needs are urgent. In general, the free-market competition of applicants to the NIH for training and education funds, while advantageous in some respects, is problematic in others. The disparities could be addressed by developing a national cancer prevention workforce plan.

\section{Forming a National Cancer Prevention Workforce Plan}

Three resources are needed to create and implement a national cancer prevention workforce plan: leadership, data, and commitment for sustained support in cancer prevention and control. Leadership is needed both to recognize the role that cancer prevention and control can play in reducing the burden of cancer and to champion the need to nurture and maintain a health workforce to sustain cancer prevention activities. Such leadership must facilitate identification of workforce needs in the field of cancer prevention and control and define the goals to address them. Leaders are also needed to ensure that sufficient resources are made available to sustain the workforce, to oversee implementation of needed changes and the evaluation of their impact, and to maintain as a priority developing and strengthening the workforce in cancer prevention and control. Leadership is needed from a variety of stakeholders, including research and clinical leaders in the field, funding agencies, and professional membership societies who have interest in developing their membership, as well as patient groups and communities who are direct consumers of cancer prevention services.

Another major building block in creating a national workforce plan is data about the cancer prevention workforce, both current workers and those in training. Metrics of the workforce would allow surveillance for emerging areas of need and prevention of workforce shortages. Without metrics for measuring and models for forecasting, it becomes impossible to determine whether the workforce will be sufficiently prepared to meet future needs in cancer prevention and control. Interviews with cancer center directors suggest that many centers may not track staffing metrics in ways that facilitate workforce monitoring [39]. Valuable information for evaluating training and education activities includes long-term career tracking of those who have received funding as well as those who did not. Such data may be available for trainees, current and former, who received funding from the NIH, but only to NIH staff. Ultimately, even the best plans are doomed if long-term commitment is not secured.

Ideally, a national cancer prevention workforce plan would include several elements. First, an inventory should be taken of existing training and educational resources in cancer prevention and control followed by a list of workforce gaps, either identified or shortly anticipated. The inventory and list would be organized by career stage and type of profession or discipline, and include topics and geographic areas for targeted resources. Next would be a plan for short-term management of the workforce, which would strengthen productive programs, reduce or eliminate unnecessary resources, and propose strategies to address shortages. A final section would describe long-term strategies for surveillance and evaluation of the workforce, anticipation of its needs, and prevention of shortages. For oversight and implementation of plans, roles for stakeholders would need to be specified - for individuals, professional societies, universities and professional schools, funding agencies, and systems at large that prepare and influence resources for developing the workforce. Preferably, efforts on behalf of the cancer prevention workforce would be performed in 
partnership with existing resources. For example, one of CChange's major initiatives is to engage the corporate sector in cancer prevention activities through employer-sponsored health plans [40]. The CEO-Roundtable on Cancer is another non-profit organization that uses an accreditation system to engage employers in sponsoring prevention activities [41]. In the end, whatever strategies are used should employ the best attributes of an open competition system, while also incorporating mechanisms to provide sufficient guidance to manage the workforce without being overly proscriptive or inflexible.

\section{Risk of the Status Quo}

The likelihood is good that the current system for sponsoring the training and education of the future workforce in cancer prevention will continue to serve, but perhaps only adequately so. NIH program officers and their advisors can use the tools they currently have available to shape training and education resources and minimize duplication of efforts; they can choose judiciously from among proposed programs winnowed through the peer-review process, and develop program announcements to direct interest to specific areas of need and interest. However, these are blunt tools for managing the workforce in cancer prevention effectively and the risk for failure is substantial when borne without the benefit of comprehensive surveillance data and guidance from regular feedback from those in the field. Existing gaps may continue to be unaddressed and new ones go unnoticed; when gaps are detected, the system may be sluggish in its response to meet workforce needs [16]. Mobilizing resources, recruiting individuals for training, and preparing them for careers in cancer prevention and control take time and effort, often years before professionals are ready to launch their careers. Thus, in the absence of a clearly articulated strategic plan that enjoys the endorsement of multiple stakeholders, our ability to train and prepare the workforce adequately to meet changing public health needs in cancer prevention and control remains in peril.

\section{Summary}

All indicators suggest that the need for efforts to prevent cancer will only increase. The numbers of individuals who can benefit from prevention services, including those already diagnosed with cancer or at risk for cancer, has grown over time. Regardless of the number or difficulty of the challenges, we should not allow ourselves to lapse into contentment with the current pace of preventing cancer. Given the amazing strides in the past 50 years against other major causes of death such as stroke, influenza, and cardiovascular disease, as well as those against HIV/AIDS in the past 30 years, progress against cancer and its prevention should be a major national and international goal. To achieve this goal, ensuring the health of the workforce in cancer prevention and control is imperative.

Acknowledgments We would like to thank all of the authors who participated in the articles that make up this supplement, as well as all of the participants from the symposium. In particular, we would like to thank Candice Collie for her unflagging efforts in organizing the symposium, publishing this article, and overseeing the entire supplement. The symposium and this supplement were funded in part by the Pfizer Medical Education Group, the National Cancer Institute (R13CA142015), and the Division of Cancer Prevention and Population Sciences at The University of Texas MD Anderson Cancer Center. The authors are supported in part by grant funds awarded to the Cancer Prevention Research Training Program at The University of Texas MD Anderson Cancer Center (R25CA57730, R25CA56452, R25DA026120, and U54CA153505). This research is supported in part by the National Institutes of Health through MD Anderson's Cancer Center Support Grant CA016672.

Conflict of Interest The authors declare that they have no conflict of interest.

\section{References}

1. Erikson C, Salsberg E, Forte G (2007) Future supply and demand of oncologists: challenges to assuring access to oncology services. J Oncol Pract 3:79-86

2. Chang S, Collie CL (2009) The future of cancer prevention: will our workforce be ready? Cancer Epidemiology Biomarkers and Prevention 18(9):2348-51

3. American Society of Clinical Oncology [Internet]. ASCO's Workforce Strategic Plan 2008 [cited 2009 Jul 1]. Available from: (http://www. asco.org/ascov2/Practice $+\&+$ Guidelines/Workforce+Initiatives).

4. Towle EL, Barr TR, Hanley A, Kosty M, Williams S, Goldstein MA (2011) Results of the ASCO study of collaborative practice arrangements. Journal of Clinical Oncology 29:3599-3600

5. Buswell LA, Ponte PR, Shulman LN (2009) Provider practice models in ambulatory oncology practice: analysis of productivity, revenue, and provider and patient satisfaction. J Oncol Pract 5:188-192

6. ASCO Senate hearing statement. (http://www.asco.org/ascov2/Prac tice $+\&+$ Guidelines/Workforce+Initiatives, accessed January 5, 2012)

7. US Department of Health and Human Services, Centers for Disease Control and Prevention. Public Health's Infrastructure: A Status Report. Prepared for the Appropriations Committee of the United States Senate; 2000.

8. Gotway Crawford CA, Summerfelt WT, Roy K, Chen Z, Meltzer DO, Thacker SB (2009) Perspectives on public health workforce research. J Public Health Management Practice 15: S5-S15

9. Johnson, Teddi Dineley. 2008. Shortage of U.S. public health workers projected to worsen: about 250,000 new workers needed. Nations Health, 38(4). American Public Health Association.

10. Cioffi JP, Lichtveld MY, Tilson H (2004) A research agenda for public health workforce development. J Public Health Management Practice 10(3):186-192

11. Smith AP, Lichtveld MY, Miner KR, Tyus SL, Gase LN (2009) A competency-based approach to expanding the cancer care workforce: proof of concept. Medsurg Nurs 18(1):38-49, quiz 50 
12. Smith AP, Lichtveld MY (2007) A competency-based approach to expanding the cancer care workforce. Medsurg Nurs 16(2):109 17, quiz 118. PMID: 17547269

13. Institute of Medicine. 2003. Professional education and training. In: Fulfilling the potential for cancer prevention and early detection, ed. Susan J. Curry, Tim Byers, Maria Hewitt; National Cancer Policy Board, 294-331. Washington, DC: National Academies Press.

14. Dajani Z, Geller AC (2008) Cancer prevention education in the United States Medical Schools: how far have we come? J Cancer Educ 23:204-208

15. Chair: Michael Goldstein, MD. (http://www.asco.org/ASCOv2/Mul tiMedia/Virtual+Meeting?\&vmview=vm_session_presentations view \& confID $=47 \&$ track $I D=30 \&$ sessionID $=2352$; accesse $\bar{d}$ Jan 2, 2012)

16. Institute of Medicine (2009) Ensuring quality cancer care through the oncology workforce: sustaining research and care in the $21 \mathrm{st}$ century: workshop summary. The National Academies Press, Washington, DC

17. Jeffe DB, Andriole DA (2011) Factors associated with American Board of Medical Specialties member board certification among US medical school graduates. JAMA 306(9):961-70

18. Funding for Cancer Training. Center for Cancer Training. National Cancer Institute website. (http://www.cancer.gov/researchandfund ing/cancertraining/outsidenci) Accessed January 3, 2012.

19. National Cancer Institute/National Institutes of Health. Fiscal Year 2010 - Fact Book. U.S. Department of Health and Human Services: NIH Publication No. 11-7772 (April 2011).

20. American Society of Clinical Oncology, Ganz PA, Kwan L, Somerfield MR, Alberts D, Garber JE, Offit K, Lippman SM (2006) The role of prevention in oncology practice: results from a 2004 survey of American Society of Clinical Oncology members. J Clin Oncol 24(18):2948-57, Epub 2006 May 15

21. Chlebowski RT, Sayre J, Frank-Stromborg M, Lillington LB (1992) Current attitudes and practice of American Society of Clinical Oncology-member clinical oncologists regarding cancer prevention and control. J Clin Oncol 10(1):164-8

22. The National Lung Screening Trial Research Team (2011) Reduced lung-cancer mortality with low-dose computed tomographic screening. N Engl J Med 365:395-409

23. Correspondence, Reduced lung-cancer mortality with CT screening, N Engl J Med 2011; 365:2035-2038 (November 24, 2011).

24. Cancer education and career development program (R25T). NCI R25T awards funded in 2010, Center for Cancer Training. National Cancer Institute website. (http://fundedresearch.cancer.gov/search/ ResultManager?fy=PUB2010\&mech=R25\&pd=Dorkina + Myrick), accessed December 11, 2011.

25. Funded grants. Cancer Prevention and Research Institute of Texas (CPRIT). (http://www.cprit.state.tx.us/funded-grants/?program= PREV\&entity $=\&$ cancersites $=A 11+$ Sites\&location $)$, accessed December 11, 2011.

26. American Society of Clinical Oncology. ASCO Initiative Developed to Address Lack of Diversity in Oncology Workforce. (http:// www.asco.org/ASCOv2/Meetings/Breast+Cancer+Symposium/ $2010+$ Breast+Cancer+Symposium+Daily+News $/$ ASCO +
Initiative + Developed + to + Address + Lack + of + Diversity + in + Oncology+Workforce), accessed 12/22/2011.

27. Conquer Cancer Foundation. Loan Repayment Program funding. (http://www.conquercancerfoundation.org/foundation/Cancer+ Professionals/Grants+\%26+Awards/Award+Recipients/Loan+ Repayment+Program), accessed 12/22/2011.

28. Lane DS (2000) A threat to the public health workforce: evidence from trends in preventive medicine certification and training. Am J Prev Med 18(1):87-96

29. Accreditation Council for Graduate Medical Education (ACGME), GME Information, Number of Programs (http://www.acgme.org/ads public/reports/accredited_programs.asp; accessed January 3, 2012).

30. Brenner S, Siu K (2009) Preventive medicine and public health residency training: federal policy and advocacy opportunities. J Public Health Management Practice 15:S33-S39

31. Collie CL, Brown AL, Liu CC, Chang S. Evaluation of State Cancer Plan Priorities, Future Workforce Needs, and the Impact of Cancer Prevention. Poster Presentation, American Association for Cancer Education, San Diego, California, October 2010, 2010.

32. American Society of Clinical Oncology. ASCO Cancer Prevention Curriculum (2007)(https://store.asco.org/eCommerce/Orders/prod uct.aspx?productId=124; accessed December 18, 2011).

33. Zon RT, Goss E, Vogel VG, Chlebowski RT, Jatoi I, Robson ME, Wollins DS, Garber JE, Brown P, Kramer BS (2009) American Society of Clinical Oncology. American Society of Clinical Oncology policy statement: the role of the oncologist in cancer prevention and risk assessment. J Clin Oncol 27(6):986-93, Epub 2008 Dec 15

34. Costanza ME, Hoople NE, Gaw VP, Stoddard AM (1993) Cancer prevention practices and continuing education needs of primary care physicians. Am J Prev Med 9(2):107-12

35. National Science Foundation. NSF science and engineering indicators 2006. (http://www.nsf.gov/statistics/seind06/c0/c0i.htm). Accessed October 25, 2008.

36. National Science Foundation. Graduate students and postdoctorates in science and engineering: Fall 2004. Available at: (http:// www.nsf.gov/statistics/nsf06325/). Accessed September 23, 2009.

37. World Health Organization (WHO). Ask the expert: Are the number of cancer cases increasing or decreasing in the world? (http://www. who.int/features/qa/15/en/index.html), accessed 12-21-11.

38. Chapman SA, Lindler V, McClory V, Nielsen C, Dyer W. Frontline workers in cancer data management: workforce analysis study of the cancer registry field. June 2006. NCRA, Alexandria, VA.

39. Center for workforce studies, Association of American Medical Colleges. Summary of workforce interviews with cancer center directors (conducted at AACI 2008 Annual Meeting). Oct 2008. (http://aaci-cancer.org/oncologyworkforce.asp), accessed, January 3, 2012.

40. C-Change, Collaborating to Conquer Cancer website, Making the Business Case: How engaging employees in preventive care can reduce healthcare costs. (http://c-changeprojects.org/MakingThe BusinessCase/Employers.asp); accessed January 3, 2012.

41. CEO Roundtable on Cancer (http://www.ceoroundtableoncancer. org/default.aspx; accessed on January 3, 2012). 